\title{
Scaling of High Harmonic Generation with Visible Driver Wavelengths
}

\author{
Chien-Jen Lai ${ }^{1}$, Giovanni Cirmi ${ }^{1,2}$, Eduardo Granados ${ }^{1,3}$, Shu-Wei Huang ${ }^{1}$, \\ Phillip Keathley ${ }^{1}$, Alexander Sell ${ }^{1}$, Kyung-Han Hong ${ }^{1}$, Jeffrey Moses ${ }^{1}$, and Franz Kärtner ${ }^{1,2}$ \\ ${ }^{1}$ Department of Electrical Engineering and Computer Science and Research Laboratory of Electronics, \\ Massachusetts Institute of Technology, 77 Massachusetts Avenue, Cambridge, MA 02139, USA \\ ${ }^{2}$ Center for Free-Electron Laser Science, DESY and University of Hamburg, Notkestraße 85, D-22607 Hamburg, Germany \\ ${ }^{3}$ IKERBASQUE, Basque Foundation for Science, 48011, Bilbao, Spain \\ cjlai@mit.edu
}

\begin{abstract}
The wavelength scaling of high harmonic generation efficiency and cutoff is studied with different visible driver wavelengths from a tunable optical parametric amplifier. A $\lambda^{-5.9}$ scaling relation for the efficiency is measured.

OCIS codes: (190.2620) Harmonic generation and mixing; (270.6620) Strong-field processes
\end{abstract}

\section{Introduction}

Because of the superb spatial and temporal coherence and the capability of ultrashort pulse generation in sub-fs regime, high harmonic generation (HHG) plays an important role in the development of coherent EUV or soft X-ray light sources. Although the spectral cutoff decreases with shorter driver wavelengths, the significant enhancement in efficiency is very beneficial to the applications in $<100 \mathrm{eV}$ regime [1]. Therefore, a shorter driver wavelength, e.g. the second harmonic of a Ti:sapphire or Yb-doped laser, can be a more efficient option for applications that don't require very high photon energy. In this paper, we experimentally demonstrate how the efficiency scales with driver wavelength between $400 \mathrm{~nm}$ and $800 \mathrm{~nm}$, a range that has not been studied systematically to our knowledge. The cutoff scaling for each different driver wavelength in helium is also demonstrated.

\section{Experimental Setup}

Fig. 1 shows our wavelength-tunable optical parametric amplification (OPA) system in the visible, which is similar to the work in Ref. [2]. It starts with a commercial Ti:sapphire amplifier delivering 6mJ, 35fs pulses at 800nm center wavelength and $1 \mathrm{kHz}$ repetition rate. $1 \%$ of the output energy is used to generate the seed in the visible range by white light generation in a sapphire plate. The remaining energy of the $800 \mathrm{~nm}$ pulses is frequency-doubled to $400 \mathrm{~nm}$ in two separated arms to pump three noncollinear OPA stages that use BBO crystals as the nonlinear media. The output wavelength of the entire OPA system is broadly tunable, depending on the noncollinear angle of the BBO crystals, from $470 \mathrm{~nm}$ to $630 \mathrm{~nm}$ with $\sim 200 \mu \mathrm{J}$ output pulse energy. The pulse duration is characterized by selfdiffraction background-free autocorrelation technique, which is measured to be between 34 and 46fs in FWHM, depending on the wavelength. The output beam has an $\mathrm{M}^{2}$ value of 1.9. The output laser pulse is delivered to the HHG chamber where the gas medium is loaded by a pulsed valve synchronized to the laser. The HHG signal is detected by an EUV spectrometer equipped with a microchannel plate (MCP) backed by a phosphor screen and a SiCCD detector.

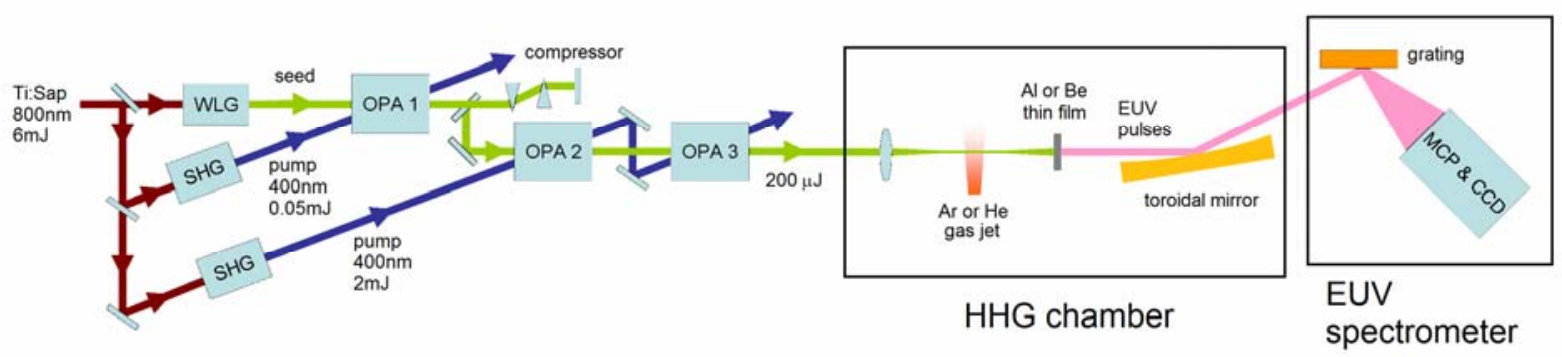

Fig. 1. Experimental setup. WLG, white light generation; SHG, second harmonic generation.

\section{High Harmonic Generation}


We use the following five different wavelengths: 400, 524, 589, 624, and 800nm, to study the HHG efficiency scaling in Ar. The 800nm and 400nm pulses are directly from the Ti:sapphire amplifier and its second harmonic respectively, and the other three wavelengths are from the OPA system described above. The harmonic spectra are measured with the EUV spectrometer. The driver pulses are focused onto a 40mbar $2 \mathrm{~mm}$-long gas jet with a fixed spot size of $25 \mu \mathrm{m}$. The spot size is controlled by adjusting the iris aperture before the HHG chamber and characterized at the focus by knife edge measurements. The pulse energies are fixed at $100 \mu \mathrm{J}$, and the intensities are fixed between $(2 \sim 3) \times 10^{14} \mathrm{~W} / \mathrm{cm}^{2}$ for all five driver wavelengths. The gas jet position is located slightly behind the laser focus and is optimized by maximizing the harmonic signal on the spectrometer. We compare the efficiencies of the $9^{\text {th }}, 13^{\text {th }}, 15^{\text {th }}, 17^{\text {th }}$, and $21^{\text {st }}$ harmonics for the five driver wavelengths respectively because these harmonics have photon energies around 32eV. Basically, the efficiencies are compared under similar experimental conditions except for the driver wavelength. The result is shown in Fig. 2(a), and the linear fitting in $\log$ scale shows a $\lambda^{-5.9}$ dependence on the driver wavelength $\lambda$. This is consistent with the $\lambda^{-(5 \sim 6)}$ scaling relation for driver wavelengths in the near and mid-IR regime [3].
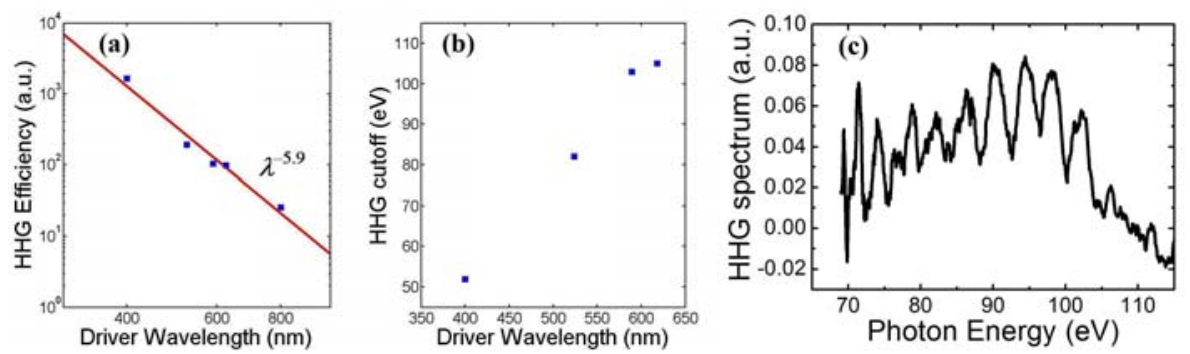

Fig. 2. (a)Efficiency scaling in Ar (b)Measured cutoff in He (c)HHG spectrum of He driven by 589nm

Since He has the highest ionization potential and the highest cutoff for a given driver wavelength among noble gases, we study the HHG cutoff of He driven by the same visible wavelengths. In the experiment, the pulse energies are fixed at $140 \mu \mathrm{J}$, which is limited by the maximum pulse energy available inside the HHG chamber. The focus spot size is adjusted to $18 \mu \mathrm{m}$ which results in an intensity of $7 \times 10^{14} \mathrm{~W} / \mathrm{cm}^{2}$. The observed cutoffs are shown in Fig. 2(b), and Fig. 2(c) shows the HHG spectrum of He around the cutoff with 589nm driver wavelength. Driver wavelengths longer than $550 \mathrm{~nm}$ are able to generate a cutoff close to $100 \mathrm{eV}$. Theoretically, the highest possible cutoff at these wavelengths should be higher than the observed cutoff shown here [1]. Being limited by the pulse energy available from our OPA system, the focusing has to be tight, and the intensity is barely enough for HHG in He. Hence, the efficiency is low, and the cutoff is limited as well mainly due to phase mismatch. However, our result still gives a lower bound of the achievable cutoff using these driver wavelengths, and the efficiency and the cutoff can be further improved with higher driver energy.

\section{Conclusion}

We systematically study how the HHG efficiency and cutoff scale with the driver wavelength over the visible regime by tuning the output wavelength of a visible OPA. A $\lambda^{-5.9}$ scaling relation of efficiency is obtained, and it provides an experimental support to the extension of the $\lambda^{-(5 \sim 6)}$ scaling law that had been found originally for the near and mid-IR regime to the visible wavelength range. We also demonstrate that the visible wavelengths are able to generate cutoff energies higher than $100 \mathrm{eV}$. These observations can be used for the development of HHG-based EUV sources needed, for example, to seed X-ray free-electron lasers.

\section{References}

[1] Edilson. L. Falcao-Filho, Chien-Jen. Lai, Kyung-Han Hong, Vasileios-Marios Gkortsas, Shu-Wei Huang, Li-Jin Chen, and Franz X. Kärtner, "Scaling of high-order harmonic efficiencies with visible wavelength drivers: A route to efficient extreme ultraviolet sources,” Appl. Phys. Lett. 97, 061107 (2010).

[2] P. Tzankov, J. Zheng, M. Mero, D. Polli, C. Manzoni, and G. Cerullo, “300 $\mu$ J noncollinear optical parametric amplifier in the visible at 1 kHz reptition rate,” Opt. Lett. 31, 3629-3631 (2006).

[3] J. Tate, T. Auguste, H. G. Muller, P. Salieres, P. Agostini, and L. F. DiMauro, "Scaling of Wave-Packet Dynamics in an Intense Midinfrared Field,” Phys. Rev. Lett. 98, 013901 (2007) 